\title{
Chronic Onset Form of Anti-HMG-CoA Reductase Myopathy
}

\author{
Noel Lorenzo-Villalba ${ }^{1}$, Emmanuel Andrès $^{1}$, Alain Meyer ${ }^{2}$ \\ ${ }^{1}$ Service de Médecine Interne, Diabète et Maladies Métaboliques, Hôpitaux Universitaires de Strasboug, France \\ ${ }^{2}$ Service de Rhumatologie, Hôpitaux Universitaires de Strasbourg, France
}

Doi: 10.12890/2021_002672 - European Journal of Case Reports in Internal Medicine - ๑ EFIM 2021

Received: 26/05/2021

Accepted: 30/05/2021

Published: 08/06/2021

How to cite this article: Lorenzo-Villalba N, Andrès E, Meyer A. Chronic onset form of anti-HMG-CoA reductase myopathy. EJCRIM 2021;8: doi:10.12890/2021_002672.

Conflicts of Interests: The authors declare there are no competing interests.

Acknowledgements: We thank Professor Lannes from the Pathology Department for her help.

This article is licensed under a Commons Attribution Non-Commercial 4.0 License

\section{ABSTRACT}

We report a case of anti-HMGCR myopathy mimicking limb-girdle muscular dystrophy in a 27-year-old male patient with no history of statin intake and presenting with a chronic onset form over 3 years. Treatment with prednisone and methotrexate was initiated with an insufficient response, so intravenous immunoglobulin was added. One year after initial treatment was started, as levels of creatine kinase (CK) were $>1000 \mathrm{U} / \mathrm{I}$, treatment with rituximab was added. Despite a 3-year delay before treatment, muscle strength improved even though CK levels remain elevated.

\section{LEARNING POINTS}

- We describe a case of anti-HMGCR myopathy mimicking limb-girdle muscular dystrophy, which resulted in delayed diagnosis and management.

- The patient's muscular strength improved but creatine kinase levels remain elevated despite comprehensive treatment.

\section{KEYWORDS}

Anti-HMG-CoA reductase, myopathy

\section{INTRODUCTION}

Autoantibodies recognizing hydroxy-3-methylglutarylcoenzyme A reductase (HMGCR) are specifically found in patients with autoimmune myopathy and are strongly associated with both a necrotizing myopathy on biopsy and a history of statin exposure ${ }^{[1]}$. The disease is defined by proximal muscle weakness, elevated serum levels of creatine kinase (CK) and autoantibodies targeting HMGCR leading to muscle fibre necrosis ${ }^{[2]}$.

\section{CASE DESCRIPTION}

We report a case of anti-HMGCR myopathy mimicking limb-girdle muscular dystrophy in a 27-year-old male patient with no history of statin intake. The patient presented with slowly progressive muscle weakness initially affecting the upper and then the lower limbs during the previous 3 years. No history of fever, rash, myalgias, arthritis or Raynaud phenomenon was reported. No previous treatment with statins was reported. The family history was irrelevant.

Physical examination showed weakness in the proximal muscles of the upper and lower limbs. Manual muscle testing in eight muscle groups showed a combined score of $131 / 150$ on a modified Kendall 10-point scale ${ }^{[3]}$. Amyotrophy of the pectoral muscles was also noted. Tabouret's sign and Gowers's sign were negative. The patient maintained the Mingazzini manoeuvre for 8 seconds and the Barré for more 
than a minute. He could do 17 leg curls in 30 seconds. He was able to drink a glass of water in 11 seconds and he walked 605 meters in 6 minutes (below the lower limit for his age). The Physician Global Activity score was 5/10, the Patient Global Activity score was 5/10, the Health Assessment Questionnaire score was 0.5/3 (mild disability) and the Extramuscular Disease Activity score was 0/10. The MYOACT score was 4/10. The evaluation was completed by the MMT12, which gave a score of 203/220, showing a deficit of strength in the ilio-psoas, gluteus medius, gluteus maximus and deltoid muscles bilaterally, and the head flexor muscles.

Blood tests showed a significantly increased serum CK level (6918 U/I) but no signs of systemic inflammation. Renal, liver and thyroid function tests were normal. Viral serology was negative. The transthoracic echocardiogram was normal and electromyography showed myogenic involvement. T2-weighted muscle MRI showed bilateral myositis of the gluteus maximus, gluteus medius, rectus femori and adductors of the thigh. A biopsy specimen was taken from the left deltoid muscle (Fig. 1). Staining showed regenerating muscle fibres but no significant inflammation.

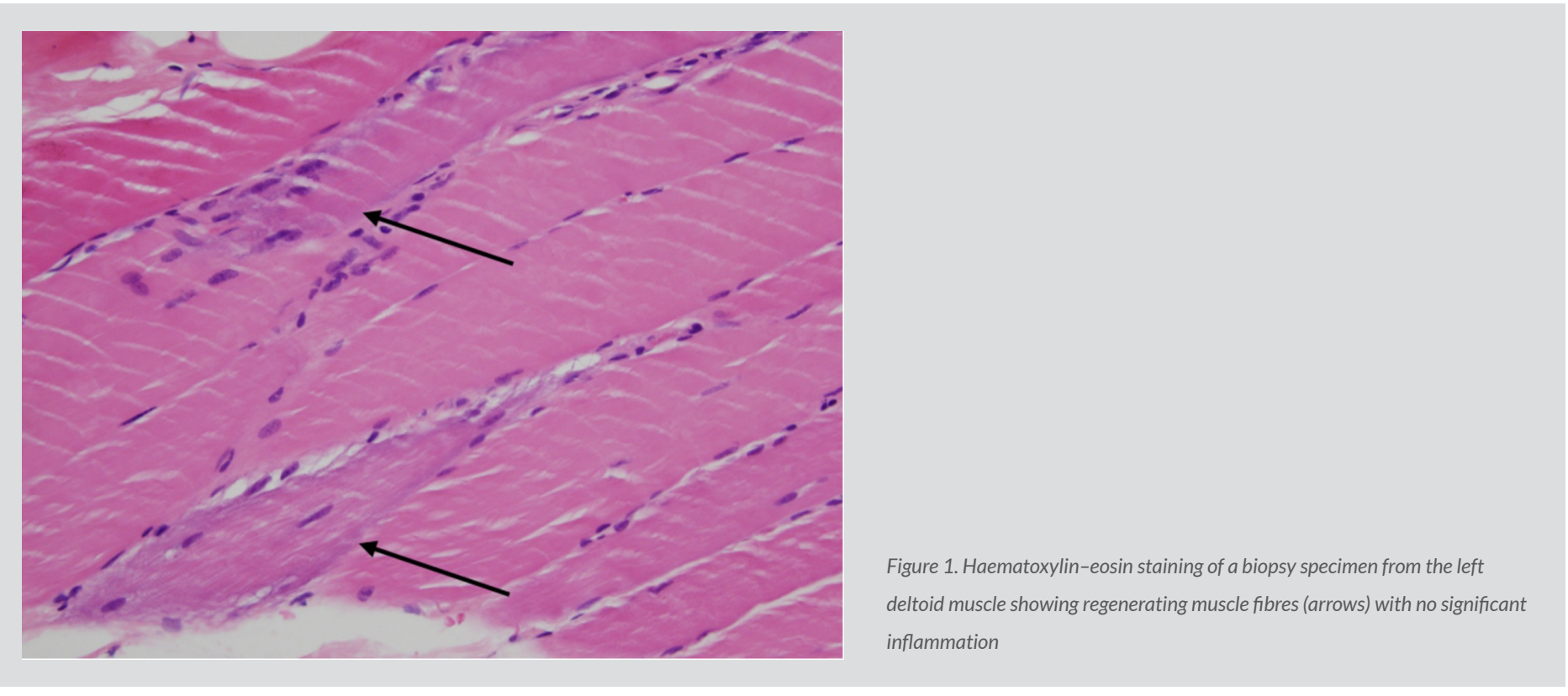

Treatment with prednisone $60 \mathrm{mg} / \mathrm{day}(1 \mathrm{mg} / \mathrm{kg} / \mathrm{day})$ and methotrexate $25 \mathrm{mg} /$ week was initiated. At 3 months, the clinical and biological response was insufficient so treatment with intravenous immunoglobulin (140 g/month) was started and methotrexate (25 mg/week subcutaneously) maintained. One year after initial treatment was started, the patient showed slight clinical improvement, but reported weakness in the lower limbs after 3 hours of prolonged standing. Blood tests still showed high levels of CK (1200 U/I). In this setting, treatment with rituximab ( $1 \mathrm{~g}$ every 15 days for a total of two doses) in accordance with ENMC recommendations was prescribed, while subcutaneous methotrexate $25 \mathrm{mg}$ and intravenous immunoglobulin $2 \mathrm{~g} / \mathrm{kg} / \mathrm{month}$ were continued.

\section{DISCUSSION}

Myopathy associated with anti-HMG-CoA reductase is characterized by subacute onset and associated with prior statin use in adults aged 50 years or older. The mechanisms underlying the development of HMG-CoA reductase autoimmunity have not been completed elucidated. Nevertheless, the class II HLA allele DRB1*11:01 has been strongly associated with the development of anti-HMG-CoA reductase autoantibodies, even in patients without known exposure to statins. In addition, the expression of HMG-CoA reductase is low in most tissues but is significantly increased when muscles are exposed to statins ${ }^{[4]}$. These elements suggest that statin-induced overexpression of HMGCoA reductase in genetically susceptible patients may cause autoimmunity against HMG-CoA reductase ${ }^{[4]}$. The presence of anti-HMG-CoA reductase autoantibodies has been described in patients who have never been prescribed statins ${ }^{[5]}$, as with our patient.

Chronic proximal muscle weakness, atrophy and necrosis of muscles fibres in young adults are hallmarks of limb-girdle muscular dystrophies, a group of genetic myopathies often requiring complex genetic testing and currently lacking effective therapies ${ }^{[6]}$. Despite showing these symptoms, and to avoid an incorrect diagnosis, our patient was tested for anti-HMGCR autoantibody ${ }^{[7]}$, which came back positive.

A chronic form of anti-HMGCR misdiagnosed as limb-girdle muscular dystrophy has been reported. Muscle response during late stage muscle damage is poor. Anti-HMGCR myopathy responds to immunomodulatory treatment ${ }^{[8]}$. Delay in treatment is a factor independently 
associated with outcome in anti-HMGCR myopathy ${ }^{[8]}$. In a previously published study, treatment regimens varied between patients based on clinical course and doctor preference, with corticosteroids (74\%), methotrexate (50\%) and intravenous immunoglobulin (39\%) the most frequently prescribed treatments ${ }^{[1]}$.

Patients with anti-HMG-CoA reductase autoantibodies who have never taken statins constitute one third of the sample in the largest cohort studied to date. These patients are younger and their myopathy is less responsive to therapy than that of statin-exposed patients ${ }^{[9]}$. In our patient, despite a 3-year delay before treatment, muscle strength improved even though CK levels remained elevated.

The chronic onset form of anti-HMG-CoA reductase myopathy is a challenging diagnosis needing a high index of suspicion.

\section{REFERENCES}

1. Tiniakou E, Pinal-Fernandez I, Lloyd TE, Albayda J, Paik J, Werner JL, et al. More severe disease and slower recovery in younger patients with anti-3-hydroxy-3-methylglutarylcoenzyme A reductase-associated autoimmune myopathy. Rheumatology (Oxford) 2017;56(5):787-794.

2. Allenbach Y, Mammen AL, Benveniste O, Stenzel W, Immune-Mediated Necrotizing Myopathies Working Group. 224th ENMC International Workshop: Clinico-seropathological classification of immune-mediated necrotizing myopathies Zandvoort, The Netherlands, 14-16 October 2016. Neuromuscul Disord 2018;28(1):87-99.

3. Rider LG, Koziol D, Giannini EH, Jain MS, Smith MR, Whitney-Mahoney K, et al. Validation of manual muscle testing and a subset of eight muscles for adult and juvenile idiopathic inflammatory myopathies. Arthritis Care Res 2010;62(4):465-472.

4. Mammen AL. Statin-associated autoimmune myopathy. N Engl J Med 2016;374(7):664-669.

5. Allenbach Y, Drouot L, Rigolet A, Charuel JL, Jouen F, Romero NB, et al. Anti-HMGCR autoantibodies in European patients with autoimmune necrotizing myopathies: inconstant exposure to statin. Medicine (Baltimore) 2014;93:150-157.

6. Thompson R, Straub V. Limb-girdle muscular dystrophies - international collaborations for translational research. Nat Rev Neurol 2016;12(5):294-309.

7. Tansley SL, Li D, Betteridge ZE, McHugh NJ. The reliability of immunoassays to detect autoantibodies in patients with myositis is dependent on autoantibody specificity. Rheumatology (Oxford) 2020;59(8):2109-2114.

8. Meyer A, Troyanov Y, Drouin J, Oligny-Longpré G, Landon-Cardinal O, Hoa S, et al. Statin-induced anti-HMGCR myopathy: successful therapeutic strategies for corticosteroidfree remission in 55 patients. Arthritis Res Ther 2020;22(1):5.

9. Werner JL, Christopher-Stine L, Ghazarian SR, Pak KS, Kus JE, Daya NR, et al. Antibody levels correlate with creatine kinase levels and strength in anti-3-hydroxy-3methylglutaryl-coenzyme A reductase-associated autoimmune myopathy. Arthritis Rheum 2012;64:4087-40893. 